\title{
A comparison between allogeneic stem cell transplantation from unmanipulated haploidentical and unrelated donors in acute leukemia
}

Simona Piemontese ${ }^{1,2^{*}}$ D, F. Ciceri ${ }^{1,2}$, M. Labopin ${ }^{2,3}$, W. Arcese ${ }^{4}$, S. Kyrcz-Krzemien ${ }^{5}$, S. Santarone ${ }^{6}$, H. Huang ${ }^{7}$, D. Beelen ${ }^{8}$, N. C. Gorin ${ }^{2,3}$, C. Craddock ${ }^{9}$, Z. Gulbas ${ }^{10}$, A. Bacigalupo ${ }^{11}$, M. Mohty ${ }^{2,3 \dagger}$, A. Nagler ${ }^{2,12 \dagger}$ and on behalf of the Acute Leukemia Working Party of the European Society for Blood and Marrow Transplantation (EBMT)

\begin{abstract}
Background: In the absence of a HLA-matched related or matched unrelated donor, allogeneic stem cell transplantation (allo-SCT) from mismatched unrelated donors or haploidentical donors are potential alternatives for patients with acute leukemia with an indication to allo-SCT. The objective of this study was to compare the outcome of allo-SCT from T cell-replete haploidentical (Haplo) versus matched (MUD 10/10) or mismatched unrelated donor at a single HLA-locus (MMUD 9/10) for patients with acute leukemia in remission.

Methods: Two hundred sixty-five adult patients with de novo acute leukemia in first or second remission that received a Haplo-SCT between January 2007 and December 2013 were compared with 2490 patients receiving a MUD 10/10 and 813 receiving a MMUD 9/10. Propensity score weighted analysis was conducted in order to control for disease risk imbalances between the groups.

Results: The weighted 3-year non-relapse mortality and relapse incidence were 29 and 30\% for Haplo, 21 and 29\% for MUD 10/10, and 29 and 25\% for MMUD 9/10, respectively. The weighted 3-year leukemia-free survival (LFS) and overall survival (OS) were 41 and 46\% for Haplo, 50 and 56\% for MUD 10/10, and 46 and 48\% for MMUD 9/10, respectively. Using weighted Cox model, both LFS and OS were significantly higher in transplants from MUD 10/10 compared from those in Haplo but not different between transplants from MMUD 9/10 and Haplo. The type of donor was not significantly associated with neither acute nor chronic graft-versus-host disease.
\end{abstract}

Conclusions: Patients with acute leukemia in remission have better outcomes if transplanted from a MUD 10/10. We did not find any significant difference in outcome between transplants from MMUD 9/10 and Haplo, suggesting that both can be equally used in the absence of a 10/10 MUD.

Key point 1: Better outcomes using fully (10/10) matched unrelated donor for allo-SCT in acute leukemia in remission.

Key point 2: Similar outcomes after allo-SCT from unmanipulated haploidentical graft or mismatched (9/10) unrelated donor in acute leukemia in remission.

\footnotetext{
* Correspondence: piemontese.simona@hsr.it

${ }^{\dagger}$ Equal contributors

${ }^{1}$ Hematology and Bone Marrow Transplant Unit, San Raffaele Scientific

Institute, Milan, Italy

${ }^{2}$ EBMT ALWP Office, Hospital Saint Antoine, Paris, France

Full list of author information is available at the end of the article
}

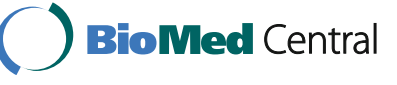

(c) The Author(s). 2017 Open Access This article is distributed under the terms of the Creative Commons Attribution 4.0 International License (http://creativecommons.org/licenses/by/4.0/), which permits unrestricted use, distribution, and reproduction in any medium, provided you give appropriate credit to the original author(s) and the source, provide a link to the Creative Commons license, and indicate if changes were made. The Creative Commons Public Domain Dedication waiver (http://creativecommons.org/publicdomain/zero/1.0/) applies to the data made available in this article, unless otherwise stated. 


\section{Background}

Allogeneic stem cell transplantation (allo-SCT) represents the only possible cure for most adult acute leukemias (AL). HLA-matched related (MRD) or unrelated donors (MUD) are usually considered the preferable donors, but they are not available for all the patients with an indication for allo-SCT. In the absence of a HLA-matched donor, allo-SCT from mismatched unrelated donors (MMUD), cord blood units (CB), or haploidentical (Haplo) donors are potential alternatives.

A Haplo donor is available for virtually all AL patients, enabling minimal delay and access to repeated stem cell (SC) donations or donor lymphocyte infusions, if needed. These are the main reasons for the increasing numbers of Haplo-SCT for AL in recent years. Unmanipulated (non ex vivo T-depleted) grafts from Haplo donors, in comparison to T-depleted ones, result in lower incidence of serious infections due to faster immune reconstitution and stronger graft versus leukemia effect [1, 2]. In addition, the introduction of more effective regimens for graft-versus-host disease (GvHD) prophylaxis for $\mathrm{T}$ replete Haplo-SCT contributed to reduce GvHD incidence and to increase the use of unmaniplated grafts for the Haplo setting [3-10].

Recently, several reports have shown comparable allo-SCT outcomes between Haplo and historical MRD, MUD, and MMUD series [11-15]. However, these are mostly but one [14] single center studies with limited number of patients $[11,12,15]$ in various disease categories and status. For these reasons, we decided to perform a large, registry-based study, using the European Society of Bone Marrow Transplantation (EBMT)-Acute Leukemia Working Party (ALWP) registry, comparing T-replete Haplo-SCT to transplants from MUD and MMUD for AL patients in first or second remission.

\section{Methods}

In order to be included in the study, the patients had to fulfill all the following criteria: age $\geq 18$ years; de novo AL; disease status at transplant: complete remission 1 (CR1) or 2 (CR2); family donor with host/donor number of HLA mismatches $\geq 2$ (Haplo), or MUD 10/10 or MMUD 9/10 (patients and donors should have HLA A, $\mathrm{B}, \mathrm{C}$, and DRB1 and DQB1 allelic typing performed); peripheral blood $(\mathrm{PB})$ or bone marrow $(\mathrm{BM})$ or both as source of SC; no ex vivo T cell depletion; and first alloSCT (previous autologous SCT was allowed). All patients underwent transplantation between January 2007 and December 2013. We were able to verify the inclusion criteria for 265 Haplo-SCT, 2490 MUD 10/10-SCT, and 813 MMUD 9/10-SCT. This was a retrospective multicenter analysis. Data were provided and approved for this study by the ALWP of the EBMT group registry.
The EBMT is a non-profit, scientific society representing more than 600 transplant centers mainly in Europe. The EBMT promotes all activity aiming to improve stem cell transplantation or cellular therapy, which includes registering all the activity relating to stem cell transplants. Data are entered, managed, and maintained in a central database with internet access; each EBMT center is represented in this database. There are no restrictions on centers for reporting data, except for those required by the law on patient consent, data confidentiality, and accuracy. Quality control measures included several independent systems: confirmation of validity of the entered data by the reporting team, selective comparison of the survey data with minimum essential data $\mathrm{A}$ (MED-A) data sets in the EBMT registry database, cross-checking with the National Registries, and regular in-house and external data audits. Since 1990, patients have provided informed consent authorizing the use of their personal information for research purposes.

\section{Definitions and statistical analysis}

The primary endpoints were leukemia-free survival (LFS) and overall survival (OS). The secondary endpoints were engraftment, acute and chronic GVHD (aGVHD and cGVHD), relapse incidence (RI), nonrelapse mortality (NRM), and graft-versus-host relapsefree survival (GRFS) [16]. LFS was defined as time to death or relapse, whichever came first. OS was defined as time to death from all causes. NRM was defined as death without evidence of relapse. Engraftment was defined as the first of three consecutive days with an absolute neutrophil count $>0.5 \times 10^{9} / \mathrm{L}$. Acute GVHD was graded according to the modified Seattle-Glucksberg criteria [17] and cGVHD according to the revised Seattle criteria [18].

We used propensity scores (PS) weighting to control for pre-treatment imbalances on observed variables. The following factors were included in the PS model: patient age, time from diagnosis to transplantation, year of transplant, diagnosis (AML versus ALL), status at transplant (CR1 versus CR2), cytogenetics group, donor/ patient CMV serology, conditioning (RIC versus MAC), and sex matching (female donor to male recipient versus other). The estimation of propensity score was performed using generalized boosted models [19].

As the study question was whether Haplo could replace $10 / 10$ or $9 / 10$ MUD, we weighted the groups receiving either MUD 10/10- and MMUD 9/10-HSCT to match the characteristics of patients receiving Haplo-SCT, by estimating the average treatment effect among the treated (ATT); Haplo-HSCT being the treated group. The ATT weights equal one for Haplo-HSCT, and it equals the ratio of the propensity score to one minus the propensity score in the two UD-HSCT groups. Therefore, UD patients that 
were significantly different from average haplo-grafted patients had a low contribution in the comparisons. We checked the balance between the groups looking to ATT weighted means. Then, we used pairwise ATTs to fit the weighted Kaplan-Meier and Cox models separately for Haplo- versus MUD 10/10-HSCT and Haplo- versus MMUD 9/10-HSCT.

The type I error rate was fixed at 0.05 for determination of factors associated with time to event. Analyses were performed using the $\mathrm{R}$ statistical software version 3.2.3 ( $\mathrm{R}$ Development Core Team, Vienna, Austria); propensity score analysis was performed using the mnps function of the Twang package and weighted analyses using the survey package [20].

\section{Results}

\section{Patients' and donors' characteristics}

Patient's and host/donors' characteristics are showed in Table 1. Regimens, stem cell source, and GvHD prophylaxis are described in Table 2. Anti-thymocyte globulin (ATG) was used for in vivo T cell depletion in 120/265 Haplo (45\%), 1457/2490 (59\%) 10/10 MUD, and 550/813 (82\%) 9/10 MMUD. Campath was used in 6/265 Haplo (1\%), 302/2490 (12\%) MUD, and 124/813 (18\%) MMUD. PT-Cy was used in 107/265 Haplo (40\%), 27/2490 (1\%) MUD, and 12/813 (1\%) MMUD. Among patients receiving a MMUD 9/10, 204 (25\%) were mismatched in locus A, 115 (14\%) in locus B, 275 (34\%) in locus C, 62 (8\%) in locus DRB1, and $157(19 \%)$ in locus DQB1. Allelic typing for locus A, B, C, DRB1, and DQB1 was available for all patients and donors.

\section{Engraftment}

The 30-day CI of engraftment was 95\% (92-97\%) for Haplo, 97\% (93-98\%) for MUD 10/10, and 92\% (8895\%) for MMUD 9/10. In weighted Cox model, CI of engraftment resulted to be lower in Haplo versus MUD $10 / 10(p=0.015)$ but not different between Haplo and MMUD 9/10 $(p=0.62)$.

\section{Non-relapse mortality, acute and chronic GvHD}

The weighted CI of NRM at 3 years was 29\% (23-34\%), 21\% (15-26\%), and 29\% (23-35\%) for Haplo , MUD 10/ 10, and MMUD 9/10 (Fig. 1a), respectively. In weighted Cox model, NRM was lower in MUD 10/10 as compared in Haplo, but not different from that in MMUD 9/10 (Table 3). The percentage of grades II-IV aGvHD was $28 \%$ (22-33\%), 25\% (18-31\%), and 27\% (21-33\%) for Haplo, MUD 10/10, and MMUD 9/10, respectively. The frequency of grades III-IV aGvHD was $10 \%$ (6-13\%) for Haplo, 7\% (3-10\%) for MUD 10/10, and 11\% (6-15\%) for MMUD 9/10. The 3-year CI of overall cGvHD and extensive cGvHD was 34\% (28-40\%) and 15\% (10-20\%), $40 \%(33-47 \%)$ and $22 \%(15-28 \%)$, and 33\% (26-39\%) and 18\% (12-23\%) for Haplo, MUD 10/10, and MMUD 9/10, respectively. Neither grades II-IV aGvHD nor chronic GvHD incidences differed between Haplo versus MUD 10/10 and Haplo versus MMUD 9/10 in weighted Cox analysis (Table 3).

\section{Relapse incidence}

The weighted CI of relapse at 3 years was 30\% (24$35 \%), 29 \%(22-35 \%)$, and $25 \%(19-31 \%)$ for Haplo, MUD 10/10, and MMUD 9/10, respectively (Fig. 1b).The type of donor was not a predictive factor for relapse in weighted Cox model (Table 3).

\section{LFS, OS, and GRFS}

The median follow-up among survivors was 34 (range, 3-84) months for Haplo, 36 (range, 1-103) for MUD 10/10, and 36 (range, 1-102) for MMUD 9/10, respectively. The weighted probability of LFS at 3 years was $41 \%$ (35-48\%), 50\% (43-58\%), and 46\% (39-53\%) for Haplo, 10/10 MUD, and 9/10 MMUD, respectively (Fig. 2).The weighted probability of 3 -year OS was $46 \%$ (40-53\%), 56\% (49-64\%), and 48\% (41-56\%) (Fig. 2).The 3 -year GRFS was 33\% (28-40\%), 36\% (29-44\%), and $34 \%$ (28-41\%) for Haplo, 10/10 MUD, and 9/10 MMUD, respectively (Fig. 2). In the weighted Cox analysis, LFS and OS resulted to be better for MUD 10/10 compared with Haplo, but no statistical difference comparing Haplo with MUD 9/10. No statistical differences were found in GRFS according to the type of donor in the weighted Cox analysis (Table 3).

\section{Discussion}

In the absence of a MRD for allo-SCT in acute leukemia, the ideal donor still remains to be determined. The current report represents a large registry study comparing the outcome between transplants from Haplo and MUD 10/10 or MMUD 9/10 for adult patients with de novo AL in remission.

As a recent ALWP-EBMT survey on unmanipulated haploidentical transplantation in AL showed a lower incidence of aGvHD in patients receiving PTCy but no significant statistical differences in either LFS or OS [21], in our study we included all the non T-depleted Haplo-SCT registered in the EBMT database.

Previous reports described worse outcomes after MMUD in comparison to MUD [22-26], and this finding was in accordance to our preliminary data and to a recent ALWP publication [27].

We did not find any differences in terms of LSF and OS in the 813 MMUD-SCT according to HLA-DQ mismatch in univariate analysis $(p=0.35)$. Therefore, we decided to compare separately MUD 10/10 and MMUD 9/10 transplants with Haplo. 
Table 1 Patients' and host/donor characteristics

\begin{tabular}{llllll}
\hline & Haplo & MUD10/10 & MMUD 9/10 & MUD 10/10 vs Haplo & MMUD 9/10 vs Haplo \\
\hline No. & 265 & 2490 & 813 & & 0.72 \\
Follow-up & $34.2(3-84)$ & $35.7(1-103)$ & $35.8(1-102)$ & 0.93 & 0.017 \\
Age & $42.8(18-75)$ & $47.0(18-76)$ & $44.8(18-71)$ & $<10-3$ & $<10-3$ \\
Year of Tx & $2011(07-13)$ & $2010(07-13)$ & $2010(07-13)$ & $<10-3$ & 0.016 \\
Diagnosis to transplant & 260 days (82-5784) & 196 days (71-5793) & $223 d(73-4452)$ & $<10-3$ & \\
Diagnosis & & & & & \\
$\quad$ AML & 176 & 1645 & 510 & & 0.28 \\
& $66 \%$ & $66 \%$ & $63 \%$ & 0.90 &
\end{tabular}

Disease status

$\begin{array}{llll}\text { CR1 } & 159 & 1901 & 580 \\ & 60 \% & 76 \% & 71 \% \\ \text { CR2 } & 106 & 589 & 233 \\ & 40 \% & 24 \% & 29 \%\end{array}$

580

$71 \%$

233

$<10-3$

0.001

$29 \%$

Patient sex

Male

Female

155

1344

442

$58 \%$

$54 \%$

1146

$46 \%$

$42 \%$

Donor sex

Male

139

$52 \%$

Female

126

$48 \%$

1782

$72 \%$

690

$28 \%$

Female to male

No

Yes

191
$72 \%$

74

$28 \%$

CMV neg to neg

No

225

$86 \%$

Yes

36

$14 \%$

Cytogenetics

$\begin{array}{llll}\text { Good } & 29 & 261 & 68 \\ & 11 \% & 10 \% & 8 \% \\ \text { Intermediate } & 168 & 1398 & 446 \\ & 63 \% & 56 \% & 55 \% \\ \text { Poor } & 68 & 831 & 299 \\ & 26 \% & 33 \% & 37 \%\end{array}$

See text for abbreviations. Cytogenetic risk at diagnosis for AML: good t(8;21), inv16 or t(16;16); poor monosomy/deletion 5 or 7, abnormalities 11q23, complex karyotype ( $\geq 3$ abnormalities); intermediate all the others. Cytogenetic risk at diagnosis for ALL: adverse $t(9 ; 22)$ or $\mathrm{t}(4 ; 11)$, intermediate all the others

\begin{tabular}{|c|c|c|c|}
\hline 2186 & 659 & & \\
\hline $88 \%$ & $82 \%$ & & \\
\hline 286 & 143 & $<10-3$ & $<10-3$ \\
\hline $12 \%$ & $18 \%$ & & \\
\hline
\end{tabular}

1673577

$69 \% \quad 73 \%$

$<10-3$

$<10-3$

$27 \%$

68

$8 \%$

446

$55 \%$

299

0.04

0.004
212 
Table 2 Conditioning regimens, stem cell source, and in vivo T cell depletion

\begin{tabular}{|c|c|c|c|c|c|}
\hline \multirow[t]{2}{*}{ No. } & Haplo & MUD10/10 & MMUD 9/10 & MUD 10/10 vs Haplo & \multirow[t]{2}{*}{ MMUD 9/10 vs Haplo } \\
\hline & 265 & 2490 & 813 & & \\
\hline \multicolumn{6}{|c|}{ Conditioning } \\
\hline \multirow[t]{2}{*}{ MAC } & 138 & 1460 & 489 & & \\
\hline & $52 \%$ & $59 \%$ & $60 \%$ & & \\
\hline \multirow[t]{2}{*}{ RIC } & 127 & 1018 & 322 & 0.03 & 0.02 \\
\hline & $48 \%$ & $41 \%$ & $40 \%$ & & \\
\hline \multicolumn{6}{|c|}{ Stem cell source } \\
\hline \multirow[t]{2}{*}{ BM } & 141 & 473 & 141 & $<10-4$ & $<10-4$ \\
\hline & $53 \%$ & $19 \%$ & $17 \%$ & & \\
\hline \multirow[t]{2}{*}{ PB } & 124 & 2017 & 672 & & \\
\hline & $47 \%$ & $81 \%$ & $83 \%$ & & \\
\hline \multicolumn{6}{|c|}{ In vivo $T$ cell depletion } \\
\hline \multirow[t]{2}{*}{ No } & 38 & 720 & 137 & $<10-4$ & $<10-4$ \\
\hline & $15 \%$ & $29 \%$ & $17 \%$ & & \\
\hline \multirow[t]{2}{*}{ ATG } & 120 & 1743 & 664 & & \\
\hline & $45 \%$ & $70 \%$ & $82 \%$ & & \\
\hline \multirow[t]{2}{*}{ Pt-Cy } & 107 & 27 & 12 & & \\
\hline & $40 \%$ & $1 \%$ & $1 \%$ & & \\
\hline
\end{tabular}

See text for abbreviations

The 30-day CI of engraftment proved to be lower in Haplo than in MUD 10/10 but not different between Haplo and MMUD 9/10. In addition to the type of donor, the number and type of chemotherapy cycles pretransplant and/or early post-transplant infections, in particular viral, could have had an impact on differences in engraftment. A lower incidence of engraftment in Haplo compared in UD was previously reported in both reduced intensity [14] and myeloablative conditioning transplants [15].

Notably, we did not find any difference in the incidence of either aGvHD $\geq$ II or cGvHD according to donor type in line with similar comparisons in literature. This was true also for grades III-IV aGvHD and extensive cGvHD. In the recent report comparing
Haplo using PT-Cy to UD from the Center for International Blood and Marrow Transplant Research (CIBMTR), the authors describe a lower incidence of acute and cGvHD in Haplo on univariate analysis [14], but most of the Haplo were performed using $\mathrm{BM}$ as stem cell source, and most of the UD using $\mathrm{PB}$ without receiving any in vivo $\mathrm{T}$ cell depletion. Of note, they did not find any difference in the 3-year cGvHD incidence according to donor type when analyzing only patients receiving $\mathrm{BM}$. The role of stem cell source as risk factor for cGvHD after Haplo-SCT remains to be determined. While some reports do not show any difference in terms of cGvHD between $\mathrm{PB}$ and $\mathrm{BM}$ in haploidentical transplantations using PTCy $[28,29]$, a recent comparison from CIBMTR

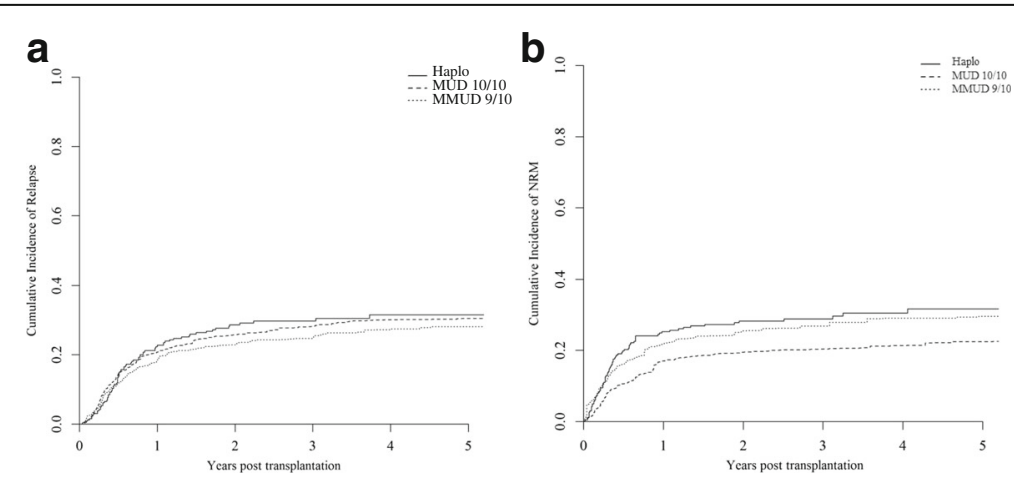

Fig. 1 Weighted Cl of relapse and non-relapse mortality according to donor type. a Weighted Cl of relapse. b Weighted Cl of NRM 
Table 3 Weighted Cox model for NRM, RI, LFS, OS, and GRFS

\begin{tabular}{|c|c|c|c|c|}
\hline \multirow[t]{2}{*}{ 3-year } & MUD 10/10 vs Haplo & $p$ & MMUD 9/10 vs Haplo & $p$ \\
\hline & \multicolumn{2}{|l|}{ HR $(95 \% \mathrm{Cl})$} & \multicolumn{2}{|l|}{ HR $(95 \% \mathrm{Cl})$} \\
\hline Day 30 PMN engraftment & $0.845(0.740-0.968)$ & 0.015 & $0.961(0.819-1.127)$ & 0.623 \\
\hline II-IV aGvHD & $0.8877(0.6777-1.163)$ & 0.387 & $1.0433(0.7716-1.411)$ & 0.783 \\
\hline III-IV aGvHD & $0.673(0.4334-1.045)$ & 0.078 & $1.184(0.7221-1.942)$ & 0.5027 \\
\hline cGVHD all & $1.0763(0.8474-1.367)$ & 0.547 & $0.9555(0.7264-1.257)$ & 0.745 \\
\hline Extensive cGvHD & $1.483(0.9890-2.224)$ & 0.057 & $1.288(0.8342-1.990)$ & 0.253 \\
\hline NRM & $0.6363(0.4838-0.8369)$ & 0.0012 & 0.9905 (0.7009-1.3997) & 0.957 \\
\hline $\mathrm{Rl}$ & $0.8624(0.6668-1.115)$ & 0.259 & $0.8426(0.6312-1.125)$ & 0.245 \\
\hline LFS & $0.7487(0.6229-0.8998)$ & 0.002 & $0.9164(0.7296-1.1511)$ & 0.45 \\
\hline OS & $0.7074(0.5824-0.8591)$ & 0.0005 & $0.9300(0.7302-1.1844)$ & 0.56 \\
\hline GRFS & $0.8804(0.7433-1.043)$ & 0.14 & $1.0313(0.8370-1.271)$ & 0.772 \\
\hline
\end{tabular}

See text for abbreviations

concludes for a high incidence of cGvHD using $\mathrm{PB}$ instead of BM [30]. Notable, in the context of UD, cGvHD has been shown to be higher using PBSC [31] especially if not using in vivo $\mathrm{T}$ cell depletion [32].

The 3-year NRM was higher using Haplo (29\%) compared with MUD 10/10 (21\%) but identical compared with MMUD (29\%), in contrast with other reports on similar comparisons where the authors did not find any difference [11-15]. This higher NRM in Haplo compared in MUD 10/10 could be in part due to the use of in vivo $\mathrm{T}$ cell depletion other than PT$\mathrm{Cy}$ in the $45 \%$ of our Haplo [33]. In addition to the type of in vivo $\mathrm{T}$ cell depletion, also, differences in conditioning regimens could explain our finding [14]. Patients' comorbidities, mostly unknown in restrospective study, could also have influenced NRM. The 3-year CI of relapse was not different between the three groups.

The lower NRM in MUD 10/10 resulted in both a higher 3-year LFS and OS compared in Haplo but no difference in GRFS. No differences in either OS,
LFS, or GRFS were observed between Haplo and MMUD 9/10. Analyzing separately patients with AML and ALL, we found no differences of GRFS in Haplo compared in MUD 10/10 and compared in MMUD 9/10, higher LFS and OS in MUD 10/10 compared in Haplo but no differences between Haplo and MMUD 9/10 in terms of LFS and OS (Additional file 1).

Based on these results, we can assert that patients with acute leukemia in remission showed better outcomes if transplanted from a MUD 10/10. We did not find any significant difference in outcome between MMUD 9/10 and Haplo, suggesting that both can be equally used in the absence of a MUD 10/10 and that other factors, such as urgency of transplant and center expertise should dictate the choice between these two alternative donor sources.

Ongoing and future prospective clinical studies, including transplants from $\mathrm{CBU}$, will ultimately be required to determine the best alternative donor for adult AL patients who lack an HLA-matched sibling one.
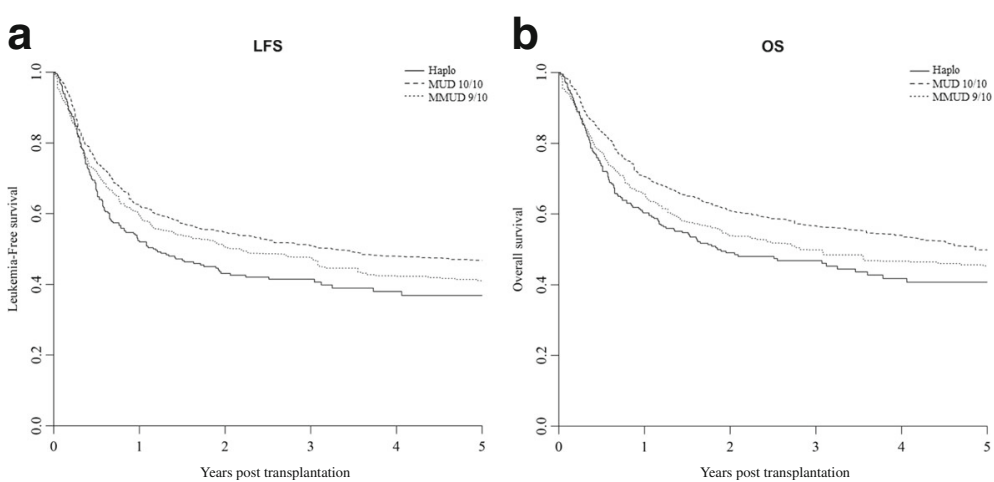

Fig. 2 Weighted probability of leukemia-free survival and overall survival according to donor type. a Weighted probability of leukemia-free survival. b Weighted probability of overall survival 


\section{Additional files}

Additional file 1: Table AML and ALL. (DOCX $18 \mathrm{~kb}$ )

Additional file 2: Participating centers' Table. (DOCX 37 kb)

\section{Abbreviations}

AL: Acute leukemia; ALL: Acute lymphoblastic leukemia; AML: Acute myeloid leukemia; ATG: Anti-thymocyte globulin; ATT: Average treatment effect among the treated; BM: Bone marrow; $\mathrm{CB}$ : Cord blood;

CMV: Cytomegalovirus; CR: Complete remission; GRFS: Graft-versus-host relapse-free survival; GVHD: Graft-versus-host disease (acute aGvHD or chronic CGVHD); HAPLO: Haploidentical donor; LFS: Leukemia-free survival; MAC: Myeloablative conditioning regimen; MMUD: Mismatched unrelated donor; MRD: Matched related donor; MUD: Matched unrelated donor; NRM: Non-relapse mortality; OS: Overall survival; PB: Peripheral blood; PMN: Polymorphonuclear cells; PS: Propensity score; PT-Cy: Post-transplant cyclophosphamide; RI: Relapse incidence; RIC: Reduced intensity conditioning regimen; SC: Stem cell; SCT: Stem cells transplantation; TX: Transplant

\section{Acknowledgements}

The authors thank all allogeneic transplantation centers of the EBMT group for reporting to the registry the data included in this analysis. A list of the participating EBMT centers appears in the Additional file 2. We thank Pr J.V. Melo for the critical reading of the manuscript.

\section{Funding}

No funding.

\section{Availability of data and materials}

Data are entered, managed, and maintained in a central database with Internet access; each EBMT center is represented in this database.

\section{Authors' contributions}

SP, ML, and FC designed the research. SP collected and checked the data and contacted the transplantation centers. SP and ML analyzed the data. SP, ML, FC, MM, AN, and N-CG wrote the paper. FC, MM, AN, AW, K-KS, SS, HH, $B D, C C, G Z$, and BA provided the data. The results of this study were partially presented as an oral presentation at the EBMT 2014 annual meeting and as a poster presentation at the ASH 2014 meeting. All authors read and approved the final manuscript.

\section{Competing interests}

The authors declare that they have no competing interests.

\section{Consent for publication}

Not applicable.

\section{Ethics approval and consent to participate}

Not applicable.

\section{Author details}

${ }^{1}$ Hematology and Bone Marrow Transplant Unit, San Raffaele Scientific Institute, Milan, Italy. 'EBMT ALWP Office, Hospital Saint Antoine, Paris, France. ${ }^{3}$ Clinical Hematology and Cellular Therapy Department, Hospital Saint Antoine, APHP, Universite Pierre et Marie Curie, INSERM UMRs 938, Paris, France. ${ }^{4}$ Stem Cell Transplant Unit, Fondazione Policlinico Tor Vergata, Tor Vergata University, Rome, Italy. ${ }^{5}$ University Department of Hematology and BMT, Medical University of Silesia, Katowice, Poland. ${ }^{6}$ Department of Hematology, Ospedale Civile, Pescara, Italy. ${ }^{7}$ Bone Marrow Transplantation Center, The First Affiliated Hospital, Zhejiang University School of Medicine, 79 Qingchun Road, Hangzhou 310003, China. ${ }^{8}$ Department of Bone Marrow Transplantation, University Hospital, Essen, Germany. ${ }^{9}$ Center for Clinical Hematology, Queen Elizabeth Hospital, Birmingham, UK. ${ }^{10}$ Bone Marrow Transplantation Department, Anadolu Medical Center Hospital, Gebze, Turkey. ${ }^{11}$ Division of Hematology II, IRCCS, San Martino University Hospital IST, Genoa, Italy. ${ }^{12}$ Division of Hematology, Chaim Sheba Medical Center, Tel-Hashomer, Israel.
Received: 26 October 2016 Accepted: 3 January 2017

Published online: 19 January 2017

\section{References}

1. O'Reilly RJ, Kernan NA, Cunningham I, et al. Allogeneic transplants depleted of T cells by soybean lectin agglutination and E-rosette depletion. Bone Marrow Transplant. 1988:3:3-6.

2. Aversa F, Tabilio A, Terenzi A, et al. Successful engraftment of T cell depleted haploidentical three loci incompatible transplants in leukemia patients by addition of recombinant human granulocyte colony stimulating factor mobilized peripheral blood progenitor cells to bone marrow inoculum. Blood. 1994;84:3948-55.

3. Ji SQ, Chen HR, Wang HX, et al. G-CSF-primed haploidentical marrow transplantation without ex vivo T cell depletion: an excellent alternative for high-risk leukemia. Bone Marrow Transplant. 2002;30:861-6.

4. Lee KH, Lee JH, Kim DY, et al. Hematopoietic cell transplantation from an HLA-mismatched familial donor is feasible without ex-vivo T-cell depletion after reduced-intensity conditioning with busulfan, fludarabine and antithymocyte globulin. Biol Blood Marrow Transplant. 2009;15:61-72.

5. Lu DP, Dong L, Wu T, et al. Conditioning including antithymocyte globulin followed by unmanipulated HLA-mismatched/haploidentical blood and marrow transplantation can achieve comparable outcomes with HLAidentical sibling transplantation. Blood. 2006;107:3065-73.

6. Luznik L, O'Donnell PV, Symons HJ, et al. HLA-haploidentical bone marrow transplantation for hematologic malignancies using nonmyeloablative conditioning and high-dose, posttransplantation cyclophosphamide. Biol Blood Marrow Transplant. 2008;14:641-50.

7. Raiola AM, Dominietto A, Ghiso A, et al. Unmanipulated haploidentical bone marrow transplantation and posttransplantation cyclophosphamide for hematologic malignancies after myeloablative conditioning. Biol Blood Marrow Transplant. 2013;19:117-22.

8. Di Bartolomeo P, Santarone S, De Angelis G, et al. Haploidentical, unmanipulated, G-CSF-primed bone marrow transplantation for patients with high-risk hematologic malignancies. Blood. 2013;121:849-57.

9. Peccatori J, Forcina A, Clerici D, et al. Sirolimus-based graft-versus-host disease prophylaxis promotes the in vivo expansion of regulatory $T$ cells and permits peripheral blood stem cell transplantation from haploidentical donors. Leukemia. 2015;29:396-405.

10. Cieri N, Greco R, Crucitti L, et al. Post-transplant cyclophosphamide and sirolimus after haploidentical hematopoietic stem cell transplantation using a treosulfan-based myeloablative conditioning and peripheral blood stem cells. Bio Blood Marrow Transplant. 2015;21:1506-14.

11. Raiola AM, Dominietto A, di Grazia C, et al. Unmanipulated haploidentical transplants compared with other alternative donors and matched sibling grafts. Biol Blood Marrow Transplant. 2014;20:1573-9.

12. Bashey A, Zhang X, Sizemore CA, et al. T-cell-replete HLA-haploidentical hematopoietic transplantation for hematologic malignancies using posttransplantation cyclophosphamide results in outcomes equivalent of contemporaneous HLA-matched related or unrelated donor transplantation. J Clin Oncol. 2013:31:1310-6.

13. Di Stasi A, Milton DR, Poon LM, et al. Similar transplantation outcomes for acute myeloid leukemia and myelodysplastic syndrome patients with haploidentical versus 10/10 human leukocyte antigen-matched unrelated and related donors. Biol Blood Marrow Transplant. 2014:20:1975-81.

14. Ciurea SO, Zhang M-J, Bacigalupo A, et al. Haploidentical transplant with post-transplant cyclophosphamide versus matched unrelated donor transplant for acute myeloid leukemia. Blood. 2015;126:1033-40.

15. Luo Y, Xiao H, Lai X, et al. T-cell-replete haploidentical HSCT with low-dose anti-T-lymphocyte globulin compared with matched sibling HSCT and unrelated HSCT. Blood. 2015;124:2735-43.

16. Ruggeri A, Labopin M, Ciceri F, et al. Definition of GvHD-free, relapse-free survival for registry-based studies: an ALWP-EBMT analysis on patients with AML in remission. Bone Marrow Transplant. 2016:51:610-1.

17. Przepiorka D, Weisdorf D, Martin P, et al. 1994 consensus conference on AGvHD grading. Bone Marrow Transplant. 1995;15:825-8.

18. Lee SJ, Vogelsang G, Flowers ME, et al. Chronic graft versus host disease. Biol Blood Marrow Transplant. 2003;9:215-33.

19. McCaffrey D, Griffin BA, Almirall D, Slaughter ME, Ramchand R, Burgette LF. A tutorial on propensity score estimation for multiple treatments using generalized boosted models. Stat Med. 2013;32:3388-414. 
20. Ridgeway G, McCaffrey D, Griffin BA, Burgette L. Twang: toolkit for weighting and analysis of non-equivalent groups. Available online at https://cran.r-project.org/web/packages/twang/vignettes/twang.pdf. Accessed 28 Apr 2016

21. Piemontese S, Ciceri F, Labopin M, et al. A survey on unmanipulated haploidentical hematopoietic stem cell transplantation in adults with acute leukemia. Leukemia. 2015;29:1069-75.

22. Finke J, Schmoor C, Lang H, et al. Matched and mismatched allogeneic stem-cell transplantation from unrelated donors using combined graftversus-host disease prophylaxis including RabbitAnti-T lymphocyte globulin. J Clin Oncol. 2003;21:506-13.

23. Flomenberg N, Baxter-Lowe LA, Confer D, et al. Impact of HLAclass I and class II high-resolution matching on outcomes of unrelated donor bone marrow transplantation: HLA-C mismatching is associated with a strong adverse effect on transplantation outcome. Blood. 2004:104:1923-30.

24. Verneris MR, Lee SJ, Ahn KW, et al. HLA Mismatch Is Associated with Worse Outcomes after Unrelated Donor Reduced-Intensity Conditioning Hematopoietic Cell Transplantation: An Analysis from the Center for International Blood and Marrow Transplant Research. Biol Blood Marrow Transplant. 2015; Epub ahead of print 1-7.

25. Petersdorf WE, Longton GM, Anasetti C, et al. Definition of HLA-DQ as a transplantation antigen. Proc Natl Acad Sci U S A. 1996;93:15358-63.

26. Loiseau P, Busson M, Balere ML, et al. HLA association with hematopoietic stem cell transplantation outcome: the number of mismatches at HLA-a, $-B$, $-C,-D R B 1$, or -DQB1 is strongly associated with overall survival. Biol Blood Marrow Transplant. 2007;13:965-74.

27. Rubio MT, Savani BN, Labopin M, et al. The impact of HLA-matching on reduced intensity conditioning regimen unrelated donor allogeneic stem cell transplantation for acute myeloid leukemia in patients above 50 years-a report from the EBMT acute leukemia working party. J Hematol Oncol. 2016 Published online Aug 3; 9(1):65.

28. Castagna L, Crocchiolo R, Furst S, et al. Bone marrow compared with peripheral stem cells for haploidentical transplantation with a nonmeloablative conditioning regimen and post-transplant cyclophosphamide. Biol Blood Marrow Transplant. 2014;20:724-9.

29. Bradstock K, Bilmon I, Kwan J, et al. Influence of stem cell source on outcomes of allogeneic reduced-intensity conditioning therapy transplants using haploidentical related donors. Biol Blood Marrow Transplant. 2015;21: 9:1641-5.

30. Bashey A, Zhang M, McCurdy SR, et al. Comparison of Peripheral Blood Stem Cells (PBSC) to Bone Marrow (BM) for T-Replete HLA-Haploidentical Donor Transplantation Using Post-Transplant Cyclophosphamide. Abstract 683 at ASH 2016: Oral presentation.

31. Lee SJ, Logan B, Westervelt P, et al. Comparison of patient-reported outcomes in 5-year survivors who received bone marrow vs peripheral blood unrelated donor transplantation: long-term follow-up of a randomized clinical trial. JAMA Oncol. 2016;2:1583-9.

32. Eapen M, Logan BR, Horowitz MM, et al. Bone marrow or peripheral blood for reduced-intensity conditioning unrelated donor transplantation. J Clin Oncol. 2015;33:364-9.

33. Ruggeri A, Sun Y, Labopin M, et al. Post-transplant cyclophosphamide versus antithymocyte-globulin as graft versus host disease prophylaxis in haploidentical transplant. Haematologica 2016. 151779. [Epub ahead of print]

\section{Submit your next manuscript to BioMed Central and we will help you at every step:}

- We accept pre-submission inquiries

- Our selector tool helps you to find the most relevant journal

- We provide round the clock customer support

- Convenient online submission

- Thorough peer review

- Inclusion in PubMed and all major indexing services

- Maximum visibility for your research

Submit your manuscript at www.biomedcentral.com/submit 